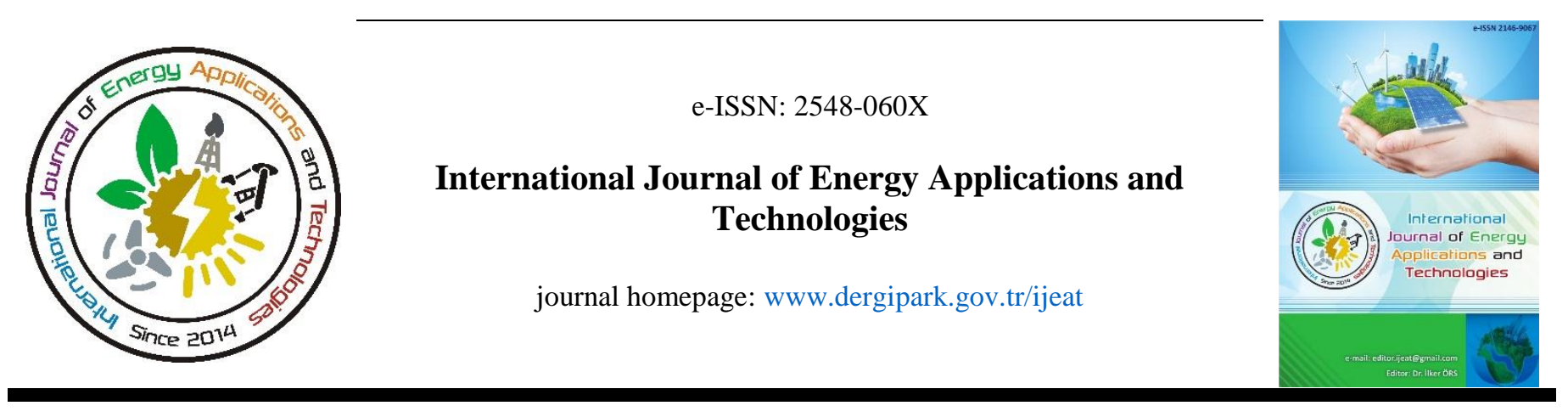

Original Research Article

\title{
Comparison of kernel functions of support vector machines: A case study for the solar cell output power prediction
}

\author{
Deogratias Nurwaha \\ Institute of Applied Pedagogy, Department of Physics and Technology, University of Burundi, Bujumbura
}

\author{
ARTICLE INFO \\ * Corresponding author \\ deogratiasnurwaha@yahoo.com \\ Received October 8, 2019 \\ Accepted February 2, 2020 \\ Published by Editorial Board \\ Members of IJEAT \\ (C) This article is distributed by \\ Turk Journal Park System under \\ the CC 4.0 terms and conditions. \\ doi: 10.31593/ijeat.630789
}

\begin{abstract}
Four kernel functions of support vector machines (SVM), namely, radial basis function, sigmoid function, linear function and polynomial function, were applied for the prediction of solar cell output power. Two types of SVM model such as epsilon-SRV and nu-SVR were chosen for each kernel function. Measured values of temperature T $\left({ }^{\circ} \mathrm{C}\right)$ and irradiance $\mathrm{E}\left(k W h \cdot \mathrm{m}^{-2}\right)$ were used as inputs and solar cell output power $\mathrm{P}(\mathrm{kW})$ was used as output. The accuracy of each kernel function was evaluated using well known statistical parameters. Radial basis function using nuSVR and polynomial function using epsilon-SVR provided similar and better results than other kernels. However, polynomial function has taken more analysis run time while radial basis function used more number of support vectors than other kernels. They may be more computationally expensive.
\end{abstract}

Keywords: Kernel function; PV output power; SVM

\section{Introduction}

To date, the interest in the use of photovoltaic (PV) energy conversion has increased worldwide. In fact, solar energy is a clean, abundant and easily available renewable energy, friendly source of energy, etc. Solar cell technology has become attractive for its potential in reducing greenhouse gas emission, consuming less fossil fuel, and providing higher penetration of renewable energy source. Also, due to its availability everywhere in the word, solar cell energy has opened up a wide range of potential applications like solar water heating, solar heating of buildings, solar distillation, solar pumping, solar drying of agricultural and power production, solar green houses, etc. [1, 2]. Other advantages of the solar cells are high reliability, minimum of cost of maintenance, long lifetime, portability, modularity, no expenditure on fuel, pollution free working, etc. However, the performance of PV systems is constantly affected by various parameters such as irradiance (E), ambient temperature (T), etc. The main difficulty of a PV controller is to predict the PV output power in order to estimate the reserve capacity. There are very little published studies available in literature regard to the modeling and prediction of solar cell output power. Also the interaction impacts of different ambient parameters on the solar cell output power and its efficiency have not been discussed enough. More recently, few studies using artificial intelligence approaches have been used to predict and model the PV power production [3-6]. Among those artificial intelligence methods, support vector machines (SVM) method has been quoted as a powerful prediction approach in many areas. Other recent studies have shown that the accuracy of SVM model depends on the chosen kernel function [7-11]. However, these functions have not yet widely explored in solar cell technology despite the main advantages that they offer. The aim of this study is to explore SVM kernel functions for based ambient parameters modeling and prediction of solar cell output power. The following SVM 
kernel functions, namely, radial basis function (RBF), sigmoid function (SIG), linear function (LIN) and polynomial function (POL), were chosen. The results provided by each kernel function will be compared.

\section{Data Collection}

Data used in this study were collected from the PV system of the Hospital of the University of Burundi. The PV system is established in the north-east of Bujumbura, the capital of Burundi. The latitude and longitude of the PV site are of $3^{\circ} 24^{\prime}$ and $29^{\circ} 21^{\prime}$, respectively. The PV system is illustrated in figure 1. Figure 2 shows the fluctuations of the temperature of the site during the year. $1920 \mathrm{PV}$ panels are installed on the area of $6300 \mathrm{~m}^{2}$. Its power capacity is of $400 \mathrm{~kW}$. The solar panels are oriented in such a way that their surfaces are more sunlight throughout the day in order to have the maximum possible energy production. Table 1 represents the specifications of the PV system. Hourly raw data including solar irradiation $\mathrm{E}\left(k W h . m^{-2}\right)$, temperature $\mathrm{T}\left({ }^{\circ} \mathrm{C}\right)$ and output power $\mathrm{P}(\mathrm{kW})$ were collected for a period of two months in sunny season (July and August) 2015. Table 2 shows the attributes and statistical properties of the collected data sets.

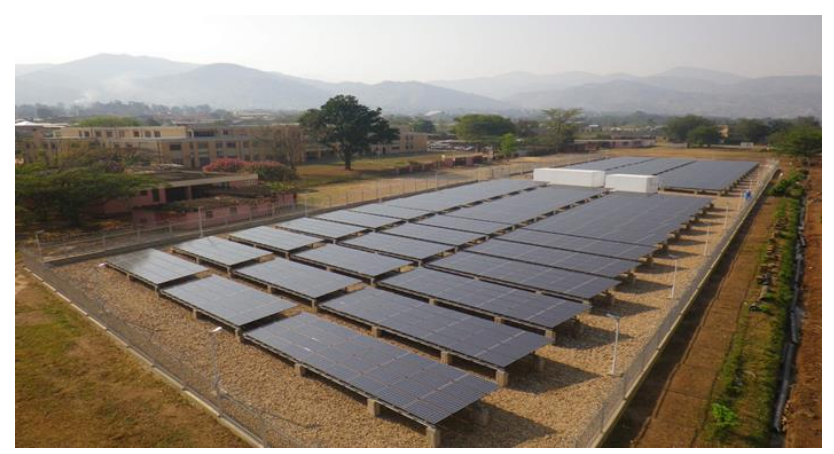

Fig. 1. PV system configuration

Table 1. Specifications of the PV system

\begin{tabular}{l|l}
\hline Number of Panels & 1920 \\
\hline Output power: $\mathrm{P}(\mathrm{kW}):$ & $400 \mathrm{~kW}$ \\
\hline Open circuit voltage: $\mathrm{V}_{\mathrm{OC}}(\mathrm{V})$ & 407.2 \\
\hline Short circuit current: $\mathrm{I}_{\mathrm{CC}}(\mathrm{A})$ & 1336.8 \\
\hline Current at maximum power: $\mathrm{I}_{\mathrm{pm}}(\mathrm{A})$ & 1221.6 \\
\hline Voltage at maximum power: $\mathrm{V}_{\mathrm{pm}}(\mathrm{V})$ & 330.4 \\
\hline Conversion efficiency: $\eta(\%)$ & 17.2 \\
\hline Area $\left(\mathrm{m}^{2}\right)$ & 6300 \\
\hline
\end{tabular}

Table 2. Statistical properties of the attributes of collected data

\begin{tabular}{ccccc}
\hline Variable & Min & Max & Mean & $\begin{array}{c}\text { Standard } \\
\text { Deviation }\end{array}$ \\
\hline $\mathbf{E}$ & 2.28 & 6.72 & 5.63 & 0.69 \\
$\mathbf{T}$ & 22.22 & 26.66 & 24.95 & 0.81 \\
$\mathbf{P}$ & 142.05 & 469.46 & 368.58 & 51.28 \\
\hline
\end{tabular}

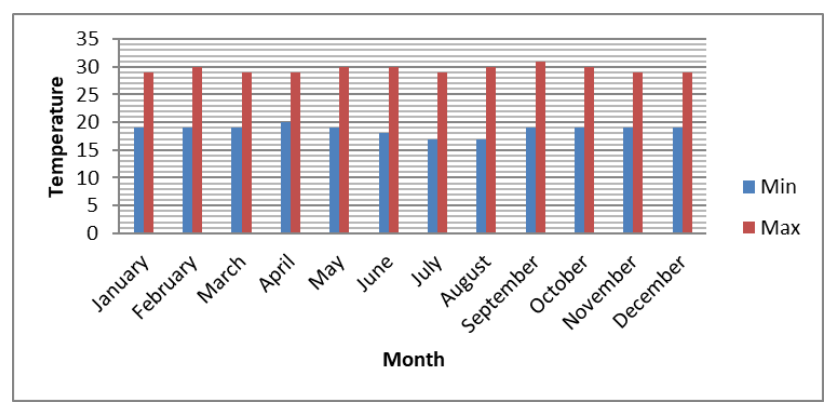

Fig. 2. PV system site's temperature fluctuations during the year

\section{Support Vector Machines}

\subsection{Introduction}

The foundations of Support Vector Machines (SVM) have been developed by Vapnik [12], and are gaining popularity due to many attractive features, and promising empirical performance. The formulation embodies the Structural Risk Minimization (SRM) principle, as opposed to the Empirical Risk Minimization (ERM) approach commonly employed within statistical learning methods. SRM minimizes an upper bound on the generalization error, as opposed to ERM which minimizes the error on the training data. It is this difference which equips SVMs with a greater potential to generalize, which is our goal in statistical learning. The SVM algorithm is based on the statistical learning theory and the VapnikChervonenkis dimension introduced by Vladimir and Alexey Chervonenkis. A support vector machine performs classification by constructing an $\mathrm{N}$-dimension hyper plane that optimally separates the data into two categories. Support vector machines models are closely related to neural networks [12]. Using a kernel function, SVM are an alternative training method for polynomial, radial basis function and multi-layer perceptron classifiers in which the weights of the network are found by solving a quadratic programming problem with linear constraints, rather than by solving a non-convex, unconstrained minimization problem as in standard neural network training. The optimal plane classifier uses only dot products between vectors in input space. So the goal of SVM modeling is to find the optimal hyperplane that separates clusters of vector in such a way that cases with one category of the target variable are on one side of the plane and cases with the other category are on the other side of the plane. The vectors near the hyperplane are the support vectors. The hyperplane can be constructed by solving a convex optimization problem that minimizing a quadratic function under linear inequality constraints. The optimization problem used to find the optimal hyperplane and the decision function used for the actual classification of vectors can be expressed in dual form from which depend only on dot products between vectors. 


\subsection{SVM kernels functions}

The most used SVM kernels are [13, 14]:

Linear (dot) Kernel: The inner product of $x$ and $y$ defines the linear kernel:

$$
K\left(x_{i}, x_{j}\right)=x_{i} \cdot x_{j}
$$

This is a linear classifier, and it should be used as a test of the nonlinearity in the training set, as well as a reference for the eventual classification improvement obtained with nonlinear kernel.

Polynomial Kernel: Polynomial kernel is a simple and efficient method for modeling nonlinear relationships:

$$
K\left(x_{i}, x_{j}\right)=\left(1+x_{i} \cdot x_{j}\right)^{d}
$$

where $\mathrm{d}$ is the degree of the polynomial. The downside of using polynomial kernels is the overfitting that might appear when the degree of the polynomial increases.

Gaussian radial Basis Function Kernel: Radial basis functions (RBF) are widely used kernels, usually in the Gaussian form:

$$
K\left(x_{i}, x_{l}\right)=\exp \left(-\frac{\|x-y\|^{2}}{2 \sigma^{2}}\right)
$$

The parameter $\sigma$ controls the shape of the separating hyperplane. It can be optimized with a suitable crossvalidation procedure.

Exponential Radial Basis Function Kernel: If discontinuities in the hyperplane are acceptable, an exponential RBF kernel is worth trying:

$$
K\left(x_{i}, x_{j}\right)=\exp \left(-\frac{\|x-y\|}{2 \sigma^{2}}\right)
$$

In some cases, this kernel requires too many support vectors Neural (Sigmoid, Tanh) Kernel: The hyperbolic tangent (tanh) function, with a sigmoid shape, is the most used transfer function for artificial neural networks. The corresponding kernel has the formula:

$$
K\left(x_{i} x_{j}\right)=\tanh \left(a x_{i} \cdot x_{j}+b\right)
$$

\section{Implementation}

In this study, we apply the type model: Support vector machine (SVM), type of analysis: regression, validation method: cross-validation, number of cross-validation folds: 10. Support vector machine kernel functions: radial basis function (RBF), sigmoid function (SIG), linear function (LIN) and polynomial function (POL), and type of SVM models: nu-SVR and epsilon-SVR. Results from each kernel function with each type of SVM model are evaluated and compared in terms of accuracies by using the well-known statistical measure parameters [15,16] namely, Root Mean
Squared Error (RMSE), Mean Absolute Error (MAE) and Mean Absolute Percentage Error (MAPE), Mean squared error (MSE), proportion of variance explained by model $\left(\mathrm{R}^{\wedge} 2\right)$, correlation between actual and predicted $(\mathrm{R})$ and coefficient of variation (CV). DTREG software [17] is used for implementation. More details on their importance can be found in [18].

\section{Results Analysis and Discussion}

The study has focused on prediction performances. A test of four SVM kernel functions has been conducted. For each kernel function, epsilon-SVR and nu-SVR as types of SVM models have been applied for comparison reasons. Table 3 shows the validation result. From the tables, it can be seen that the four kernels give results with a litter or lager difference. Optimization parameters have been chosen and are shown in table 5. But to judge the overall accuracy of each kernel, validation results must be analyzed and compared. This judgment can be easily made from table 3 or from figures 3-13.

For $\mathrm{RBF}$, the best performances, $\mathrm{CV}=0.06, \mathrm{NMSE}=0.23$, $\mathrm{MSE}=621.61, \mathrm{RMSE}=24.94, \mathrm{MAE}=20.73, \mathrm{MAPE}=5.86$, $\mathrm{R}^{\wedge} 2=76.36, \mathrm{R}=0.87$, and $\mathrm{ART}=3.04 \mathrm{~s}$ were provided by nuSVR as type of SVM model while the worst results were found by using $\varepsilon$-SVR as type of SVM model.

For SIG, the best results, $\mathrm{CV}=0.08, \quad \mathrm{RMSE}=32.84$, $\mathrm{MAPE}=7.64, \mathrm{ART}=5.43$ were provided by using nu-SVR type. Both $\varepsilon-S V R$ and nu-SVR provided similar values of NMSE, MSE, and MAE. $\mathrm{R}^{\wedge} 2$ and $\mathrm{R}$ bad results were found for the two types.

For LIN, Both $\varepsilon-S V R$ and nu-SVR provided similar values as it can be seen from table 3 .

For POL, Both $\varepsilon-S V R$ and nu-SVR provided similar values except for art values where ART $=586.47 \mathrm{~s}$ for $\varepsilon$-SVR and $\mathrm{ART}=188.4 \mathrm{~s}$ for nu-SVR. In the meanwhile, the overall bad results were provided by $\mathrm{RBF}$ and POL kernels using epsilon-SVR as type of SVM model. From the above observations, main conclusion can be drawn that accuracy of SVM kernel functions depends at less or great degree on the type of SVM model used. It was observed that POL kernel takes more analysis run time than other kernels. RBF and POL kernels use more number of support vectors than other kernels. Table 4 and figure 13 show the importance of temperature on solar cell power. From the table, one can see that the temperature contribution is significant and depends on the kernel and type of SVM model used.

KF: Kernel function; MTVID: Mean target value of input data; MTVPD: Mean target value of predicted data; CV: coefficient of variation; NMSE: Normalized mean square error, 
Table 3. Analysis of validation results

\begin{tabular}{|l|c|c|c|c|c|c|c|c|}
\hline $\begin{array}{l}\text { Statistical } \\
\text { parameter }\end{array}$ & \multicolumn{3}{|c|}{ KF( epsilon-SVR) } & \multicolumn{4}{c|}{ KF(Nu-SVR) } \\
\hline & RBF & SIG & LIN & POL & RBF & SIG & LIN & POL \\
\hline MTVID & 368.58 & 368.58 & 368.58 & 368.58 & 368.58 & 368.58 & 368.58 & 368.58 \\
\hline MTVPD & 370.98 & 372.24 & 368.29 & 372.67 & 367.32 & 370.08 & 368.45 & 369.59 \\
\hline CV & 0.12 & 0.09 & 0.08 & 0.06 & 0.06 & 0.08 & 0.08 & 0.07 \\
\hline NMSE & 0.87 & 0.41 & 0.39 & 0.24 & 0.23 & 0.41 & 0.39 & 0.27 \\
\hline MSE & 2295.52 & 1101.21 & 1028.07 & 647.50 & 621.61 & 1078.92 & 1044.63 & 717.41 \\
\hline RMSE & 47.91 & 33.18 & 32.06 & 25.44 & 24.93 & 32.84 & 32.32 & 26.78 \\
\hline MAE & 23.74 & $26 . .43$ & 25.36 & 19.62 & 20.73 & 26.19 & 25.48 & 21.30 \\
\hline MAPE & 8.89 & 7.8 & 7.35 & 5.65 & 5.86 & 7.64 & 7.44 & 6.37 \\
\hline R`2 & 12.73 & 58.13 & 60.91 & 75.38 & 76.36 & 58.98 & 60.28 & 72.72 \\
\hline R & 0.54 & 0.76 & 0.78 & 0.87 & 0.87 & 0.76 & 0.77 & 0.85 \\
\hline ART & 7.89 & 10.34 & 0.37 & 567.47 & 3.04 & 5.43 & 0.37 & 188.4 \\
\hline
\end{tabular}

ART: analysis of run time (seconds)

Table 4. Computed importance $(\%)$ of each parameter

\begin{tabular}{|l|c|c|c|c|c|c|c|c|}
\hline Variable & \multicolumn{9}{|c|}{ Importance (\%) } \\
\hline & \multicolumn{9}{|c|}{ KF epsilon-SVR } & \multicolumn{4}{c|}{ KF(NU-SVR } \\
\hline & RBF & SIG & LIN & POL & RBF & SIG & LIN & POL \\
\hline E & 100 & 100 & 100 & 100 & 100 & 100 & 100 & 100 \\
\hline T & 94.293 & 10.611 & 15.352 & 84.487 & 37.623 & 13.368 & 15.352 & 45.249 \\
\hline
\end{tabular}

Table 5. Analysis of optimization parameters

\begin{tabular}{|c|c|c|c|c|c|c|c|c|}
\hline OP & \multicolumn{4}{|c|}{ KF( epsilon-SVR) } & \multicolumn{4}{|c|}{ KF(NU-SVR) } \\
\hline & RBF & SIG & LIN & POL & RBF & SIG & LIN & POL \\
\hline$\varepsilon$ & 0.001 & 0.001 & 0.001 & 0.001 & 0.001 & 0.001 & 0.001 & 0.001 \\
\hline $\mathbf{C}$ & 14999.0494 & 6467.66824 & 2864.10608 & 12.2583245 & 13494.7685 & 5937.44367 & 10285.7194 & 1.10717318 \\
\hline$y$ & 4.62078545 & 0.11231402 & - & 50 & 0.32644013 & 0.08937303 & & 15.0266519 \\
\hline $\mathbf{P}$ & 2.29057834 & 39.8093199 & 35.4161593 & 0.00215443 & - & - & - & \\
\hline $\mathrm{Nu}$ & & & & & 0.14697918 & 0.19420658 & 0.17954782 & 0.22822156 \\
\hline NSV & 57 & 9 & 12 & 62 & 13 & 14 & 13 & 19 \\
\hline Coef0 & - & 0 & 0 & 0.59948425 & & 0 & - & 100 \\
\hline
\end{tabular}

OP: optimization parameters; y: gamma; $\varepsilon$ : epsilon; NSV: number of support vectors

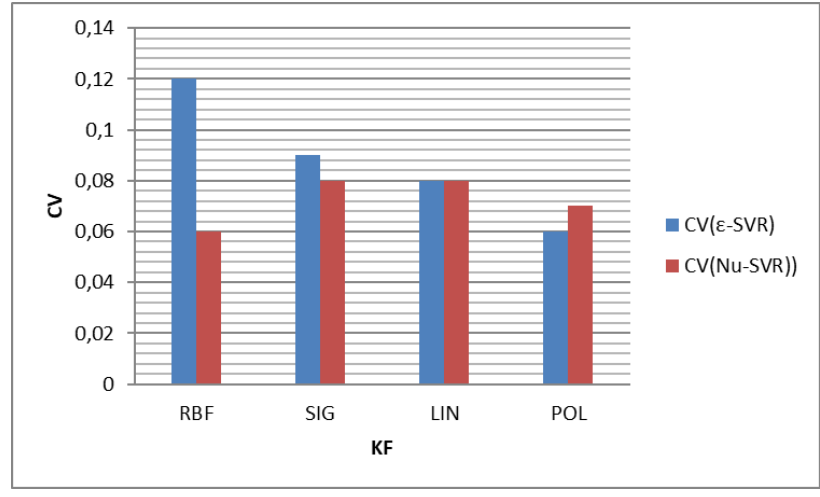

Fig. 3. Histograms of CV for different kernel functions

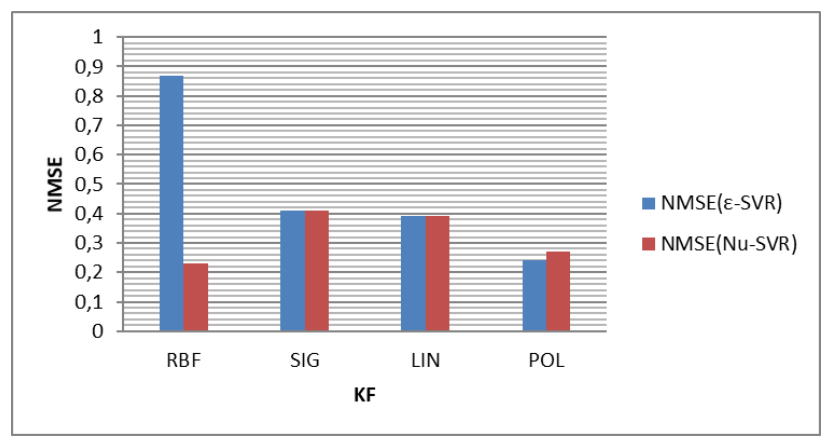

Fig. 4. Histograms of NMSE for different kernel functions

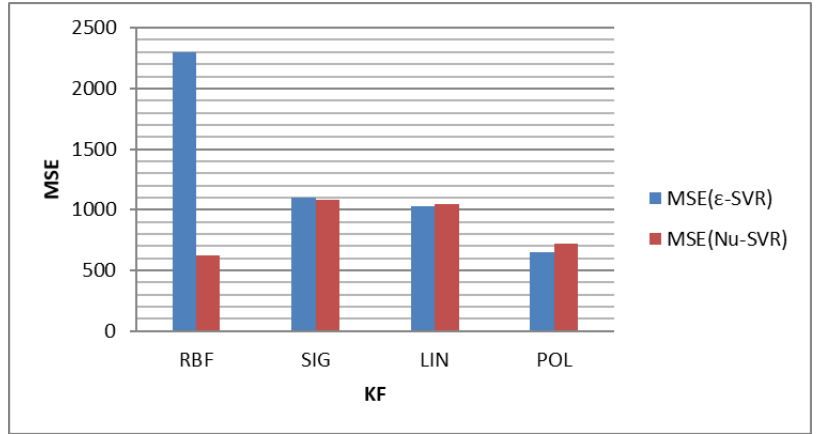

Fig. 5. Histograms of MSE for different kernel functions

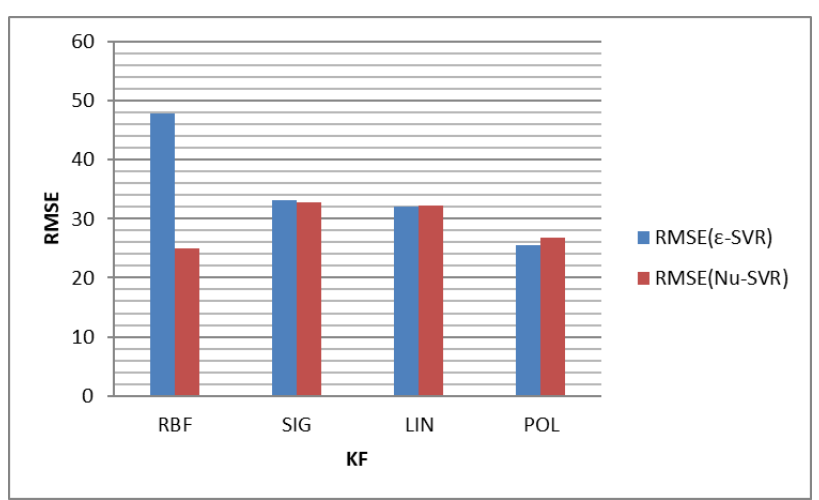

Fig. 6. Histograms of RMSE for different kernel functions 

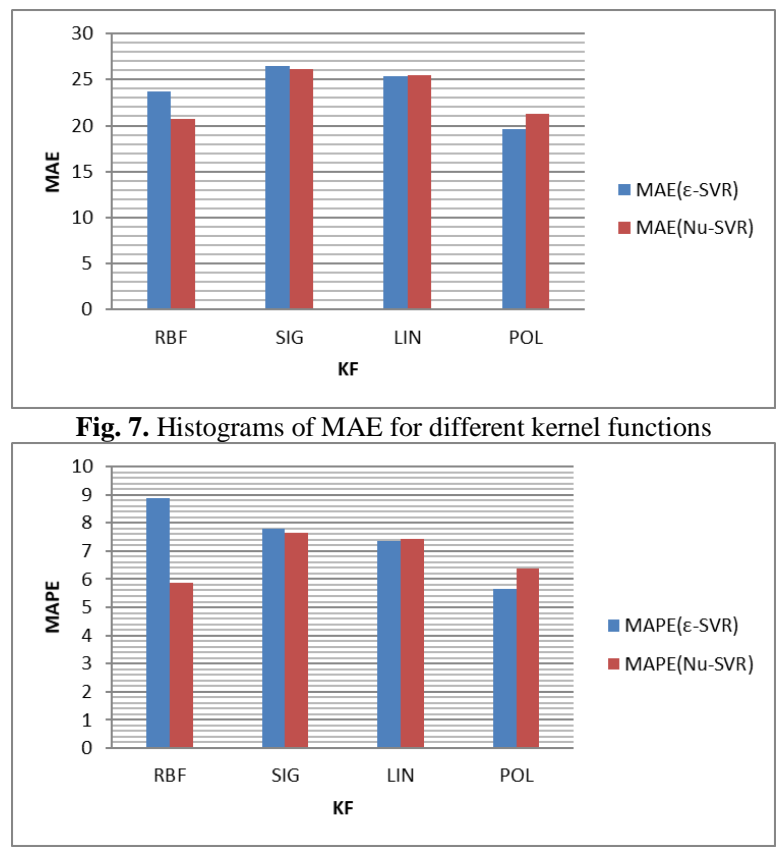

Fig. 8 .Histograms of MAPE for different kernel functions

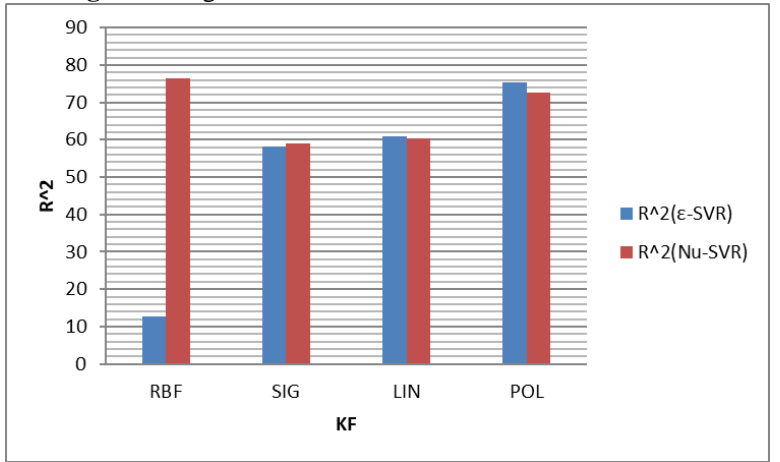

Fig. 9. Histograms of $\mathrm{R}^{\wedge} 2$ for different kernel functions

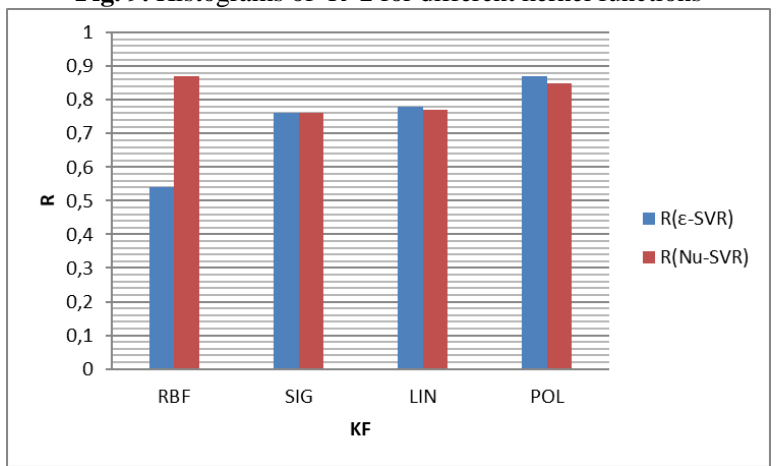

Fig. 10. Histograms of R for different kernel functions

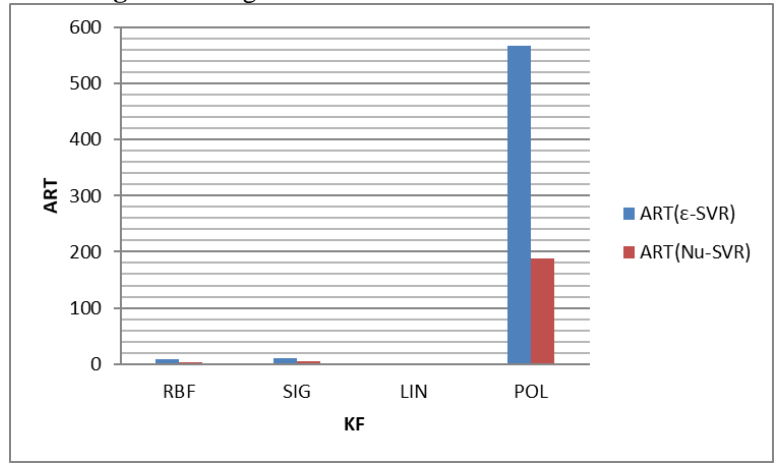

Fig. 11. Histograms of ART for different kernel functions

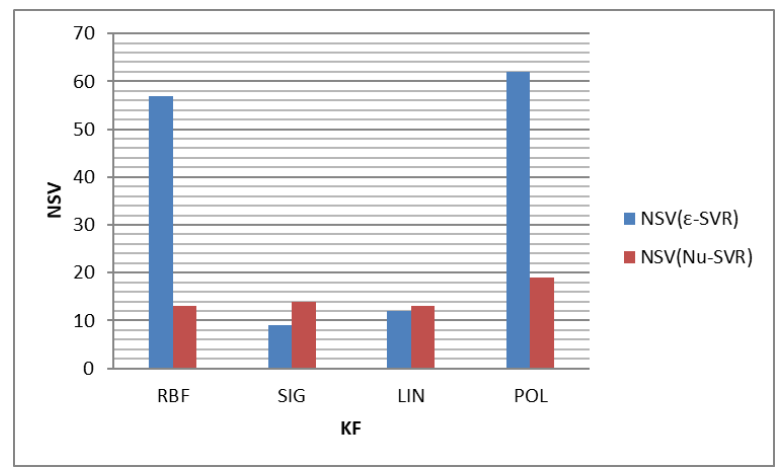

Fig. 12. Histograms of NSV for different kernel functions

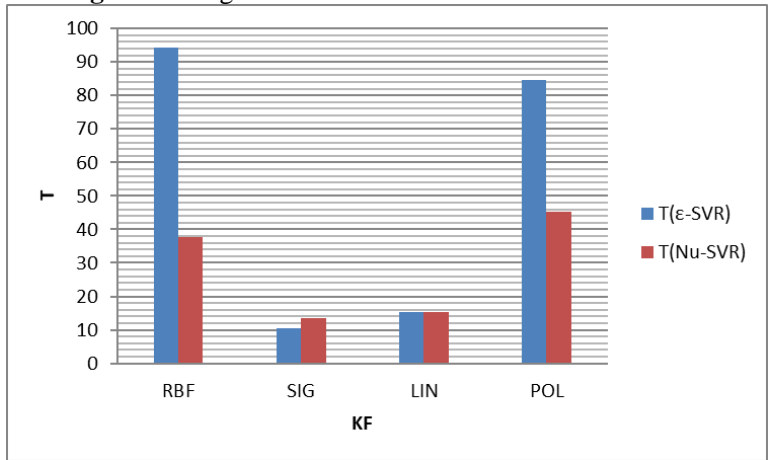

Fig. 13. Importance of temperature as contributor to the solar cell output power

\section{Conclusions}

The study investigated the prediction of solar cell output power using different SVM kernel functions. The SVM kernel functions have been successfully applied to a wide range of predictive problems. Predictive accuracy of each SVM kernel function has been evaluated using statistical metrics. The results showed that the SVM kernel functions provided good and similar performance. It was also demonstrated that accuracy of SVM kernel functions depends at less or great degree on the type of SVM model used. The importance of the type of SVM model in performing the output has been depicted. Roles played by the optimization parameters, analysis run time and number of support vectors were also illustrated. The overall results indicated that SVM kernel functions could provide a potential approach for prediction of PV output power. The main advantage of the explored methods is that the obtained results could allow the PV system operator to estimate the capacity of energy reserve. This advantage is not yet widely explored in controlling solar cell output energy. However, the analysis of different results provided by each kernel function suggested that further studies in the same aspect are still needed and compared in order to assess the acceptable accuracy of each SVM kernel function. 


\section{References}

[1] R., BoroumandJazi, G., Mekhlif, S., Jameel, M., 2012, "Exergy analysis of solar energy applications", Renewable and Sustainable Energy Reviews, 16(1)), 350-356.

[2] Sopori, B., 2002, "Silicon Solar-Cell Processing for Minimizing the Influence of Impurities and Defects", Journal of Electronic Materials, 31,972-980.

[3] Hossain, R., Maung, A., Than O,. Shawkat, A., 2013," Hybrid Prediction Method for Solar Power Using Different Computational Intelligence Algorithms",Smart Grid and Renewable Energy, 4,7687.

[4] Soteris, A., Kalogirou Şencan, A., 2010, "Artificial Intelligence Techniques in Solar Energy Applications", www.intechopen, 315-340.

[5] Hussein, A., Kazem, Jabar, H., Yousif, Miqdam T Chaichan, 2016, "Modelling of Daily Solar Energy System Prediction using Support Vector Machine for Oman", International Journal of Applied Engineering Research,11(20), 10166-10172.

[6] Anuwar, F., Omar, A., 2016," Future Solar Irradiance Prediction using Least Square Support Vector Machine", International Journal on Advanced Science Engineering Information Technology, 6(4), 513-520.

[7] Yekkehkhany, B., Safari, A., Homayouni S., Hasanlou, M., 2014," A Comparison Study of Different Kernel Functions for SVM-based Classification of Multitemporal Polarimetry SAR Data", The International Archives of the Photogrammetry, Remote Sensing and Spatial Information Sciences, Volume XL-2/W3, The 1 st ISPRS International Conference on Geospatial Information Research, 15-17, Tehran, Iran.

[8] Supriya P., Deepak S., 2015, "Comparison of Various Kernels of Support Vector Machine", International Journal for Research in Applied Science \& Engineering Technology, 3 (7),.532-536.

[9] Baudat, G., Anouar, F., 2003, "Feature vector selection and projection using kernels", Neurocomputing, 55(12), 21-38.

[10] Hong, Z., Haibin, L., Xingjian, L., Tong R., 2018,” A Multiple Kernel Learning Approach for Air Quality Prediction", ID 3506394.

[11] Yin,W., Cho,J., Kai,W., Michael, R., Chih,J., 2010, Training and Testing Low-degree Polynomial Data Mappings via Linear SVM,11, 1471-1490.

[12] Corinna, C., Vladimir, V., 1995, "Support-Vector Networks, Machine Learning”, 20, 273-297.

[13] Shawe, J.; Cristianini, N., 2004," Kernel Methods for Pattern Analysis", Cambridge University Press.
[14] Thomas, H., Bernhard, S., Alexande, R., Smola, J., 2008," Kernel Methods in Machine Learning", The Annals of Statistics, 36(3), 1171-1220.

[15] Rob J., Koehler B., 2006 "Another look at measures of forecast accuracy", International Journal of Forecasting, 22(4), 679-688.

[16] Willmott C., Matsuura, K., 2006, "On the use of dimensioned measures of error to evaluate the performance of spatial interpolators", International Journal of Geographical Information Science, 20(1), 89-102.

[17] Phillip H. S., 2014, DTREG, "Predictive Model Software".

[18] Alaa T., 2019, "Parameter investigation of support vector machine classifier with kernel functions", Knowledge and Information Systems. 